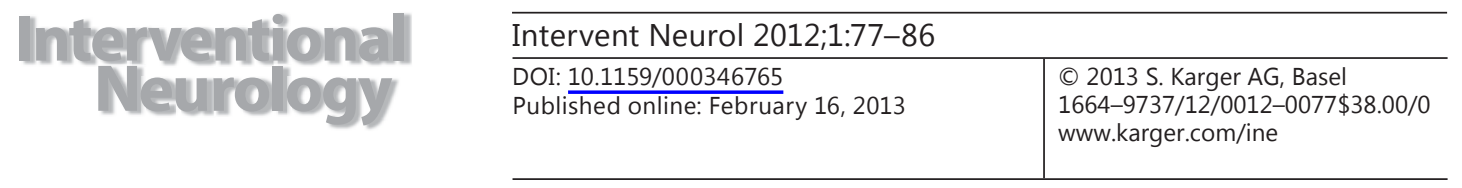

\title{
Neuroimaging Diagnosis and the Collateral Circulation in Moyamoya Disease
}

\author{
Wenhua Liu Gelin Xu Xinfeng Liu \\ Department of Neurology, Jinling Hospital, Nanjing University School of Medicine, \\ Nanjing, China
}

\section{Key Words}

Angiography · Collateral blood flow · Fluid-attenuated inversion recovery · Hyperintense vessels · Moyamoya disease

\begin{abstract}
Moyamoya disease (MMD) is an uncommon cerebrovascular disease that is characterized by progressive stenosis of the terminal portion of the internal carotid artery and its main branches, which is accompanied by the development of dilated, fragile collateral vessels at the base of the brain. This review will present different neuroimaging modalities for the diagnosis of MMD. Importantly, we will discuss the role of hyperintense vessels on fluid-attenuated inversion recovery images and their contribution to the evaluation of collateral patterns in MMD patients. Additionally, this review will summarize these common collateral patterns of MMD assessed by conventional cerebral angiography, and the associations of these angiographic collateral patterns with cerebrovascular lesions, including ischemia and hemorrhage will also be reviewed.

Copyright (c) 2013 S. Karger AG, Basel
\end{abstract}

\section{Introduction}

Moyamoya disease (MMD) is a chronic, occlusive cerebrovascular disease with bilateral stenosis or occlusion of the terminal portion of the internal carotid arteries (ICA) and/or the proximal portions of the anterior (ACA) and middle cerebral arteries (MCA) [1, 2]. Conventional cerebral angiography is the most reliable diagnostic modality for MMD. However, other modalities, such as computed tomography (CT), T2-weighted magnetic resonance imaging 
Fig. 1. CT scans and angiograms showing neuroimaging characteristics of MMD patients. a CT scan of cerebral infarction and atrophy in the right territory of the MCA. b Angiogram of the terminal occlusion of the ICA with netlike vessels. c CT scan of a left caudate nucleus hematoma and a right anterior watershed zone. d, e Angiograms of the terminal occlusion of the ICA and the moyamoya vessels in the basal ganglia.
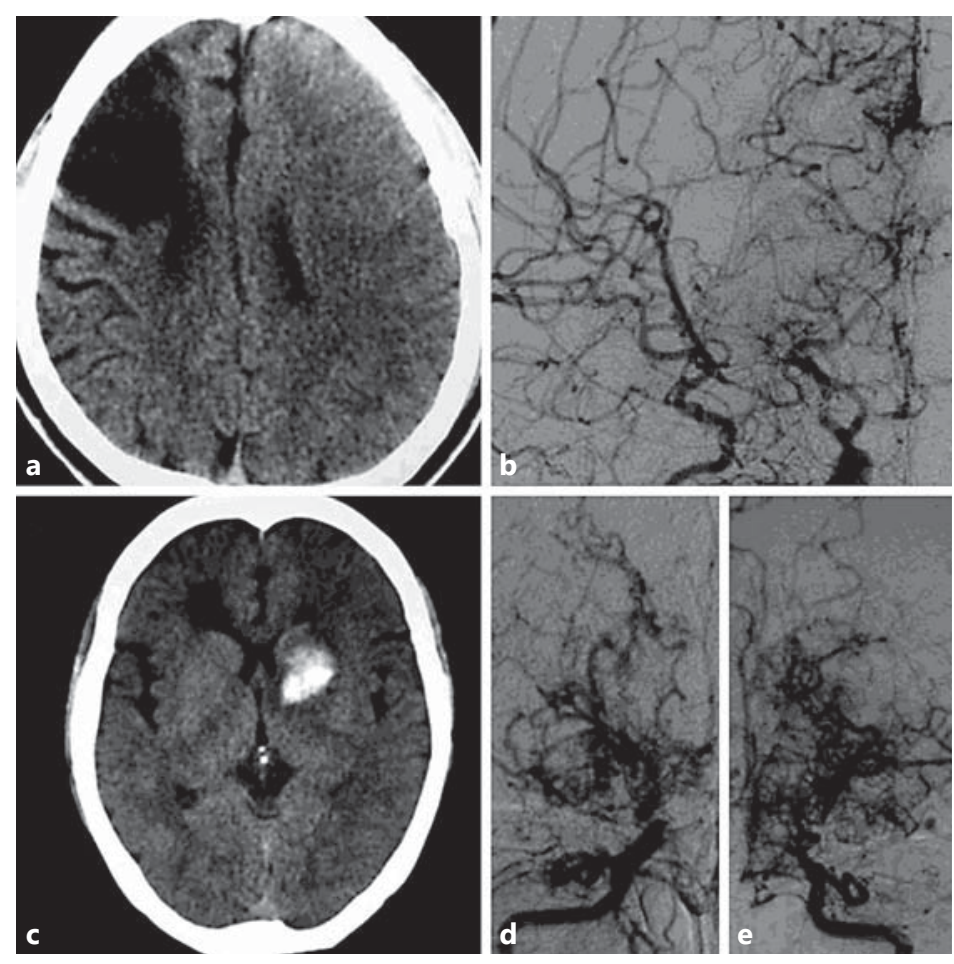

(MRI), hyperintense vessels (HV) on fluid-attenuated inversion recovery (FLAIR)-MRI, and magnetic resonance angiography (MRA) and CT angiography (CTA), also provide important information for the diagnosis of MMD. In the diagnosis of MMD, these neuroimaging modalities develop their own characteristics, and it is beneficial to master these characteristics for an early diagnosis of MMD. Importantly, we will present the role of HV on FLAIR images and their contribution to the evaluation of patterns of the collateral circulation in patients with MMD. Additionally, these common collateral patterns of MMD assessed by conventional cerebral angiography and the associations of these angiographic collateral patterns with cerebrovascular lesions, including ischemia and hemorrhage, will also be reviewed.

\section{Neuroimaging Diagnosis}

\section{Computed Tomography}

It is well known that CT cannot offer direct diagnostic information for moyamoya vessels. However, cerebral infarction and cerebral atrophy are visualized using CT in advanced stages of MMD (fig. 1a). Additionally, CT can depict simultaneously ischemic and hemorrhagic lesions of MMD (fig. 1c). CT is the most convenient neuroimaging modality that is available in most hospitals and can be performed even at midnight. Therefore, CT is used as the first diagnostic tool in most cases of MMD.

\section{Magnetic Resonance Imaging}

T2-Weighted MRI

T2-weighted-MRI, a radiation-free and noninvasive diagnostic technique, has been introduced according to the guidelines for the diagnosis of MMD in Japan by the Japanese Ministry of Health and Welfare $[3,4]$. The flow voids of large arteries due to occlusion of the circle of 

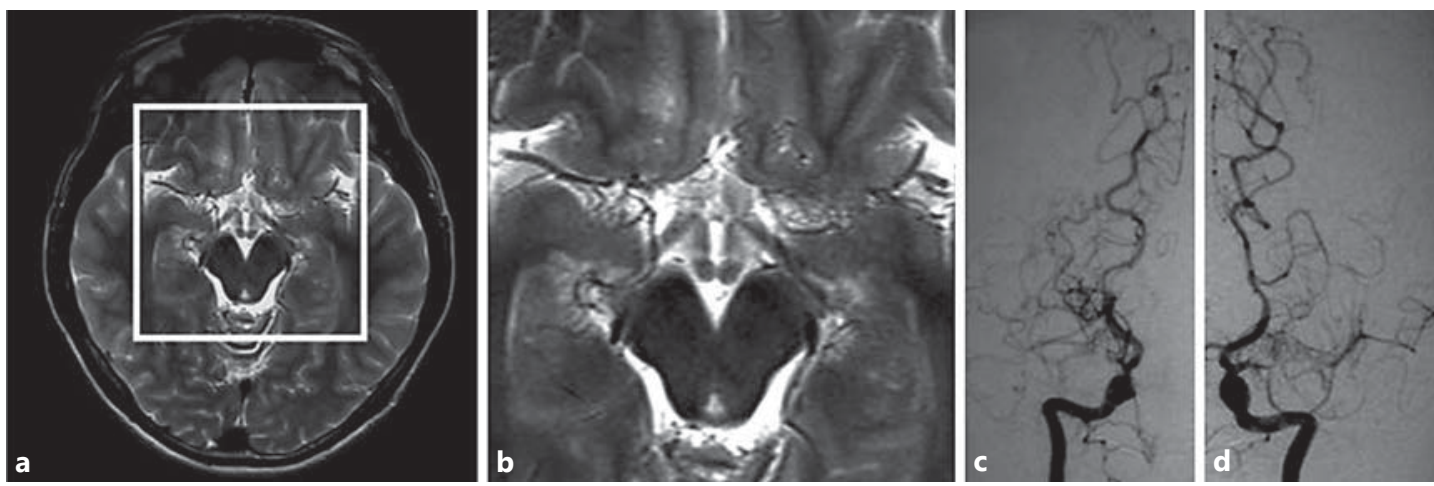

Fig. 2. T2-weighted MRI modality showing neuroimaging characteristics of MMD patients. a The steno-occlusive lesion of the circle of Willis is well disclosed in axial T2-weighted images at the level of the basal cistern. $\mathbf{b}$ Enlarged image of the inset in $\mathbf{a}$; the M1 segment of the MCA is not identified, and small signal voids corresponding to moyamoya vessels are visualized. $\mathbf{c}$, $\mathbf{d}$ Angiograms showing the terminal occlusion of the ICA and the moyamoya vessels in the basal ganglia.
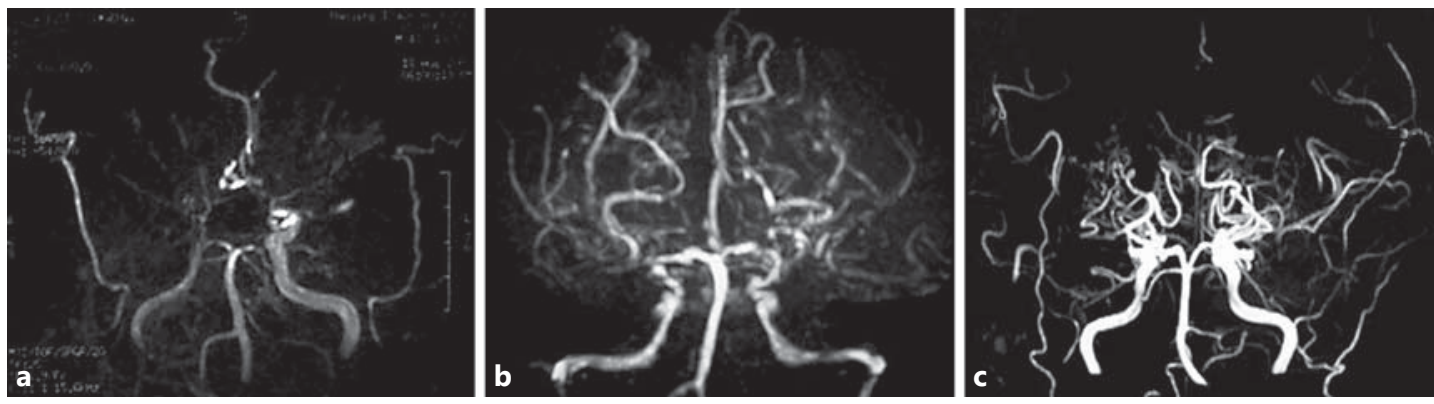

Fig. 3. MRA modalities with different tesla scanners present neuroimaging characteristics of MMD patients. a A 0.5-tesla scanner shows a steno-occlusive lesion of the circle of Willis. b A 1.5-tesla MRA established a diagnosis of MMD with unclear intra- and extracranial collateral pathway. c A 3.0-tesla machine helps to detect some small moyamoya vessels and possible collateral pathways for MMD.

Willis disappeared, and numerous flow voids appeared in the basal cistern and basal ganglia referring to the augmented vascular network at those sites. Because the black vascular flow voids are obviously in contrast to the bright cerebrospinal fluid, these findings are best shown on T2-weighted images in patients with MMD (fig. 2a, b).

\section{Magnetic Resonance Angiography}

It has been demonstrated that the neuroimaging modality of MRA is reliable in the diagnosis of MMD [4]. However, it must be borne in mind that the quality of MRA is strongly dependent on the strength of the static magnetic field, and a 1.5-tesla scanner is the minimum standard for a reliable diagnosis of MMD (fig. 3b). A 3.0-tesla MRA (fig. 3c) can be useful to detect small moyamoya vessels that are barely depicted with $0.5 \mathrm{~T}$ (fig. 3a). However, a disadvantage of MRA is that it does not provide direct information on the collateral pathways for MMD, except for 3.0-tesla MRA [5, 6].

HV on FLAIR-MRI

HV on FLAIR MRI refer to focal hyperintensity. HV can be frequently observed near the cerebral surface along the cortical sulci in patients with ischemic stroke [7-10]. HV can nonin- 

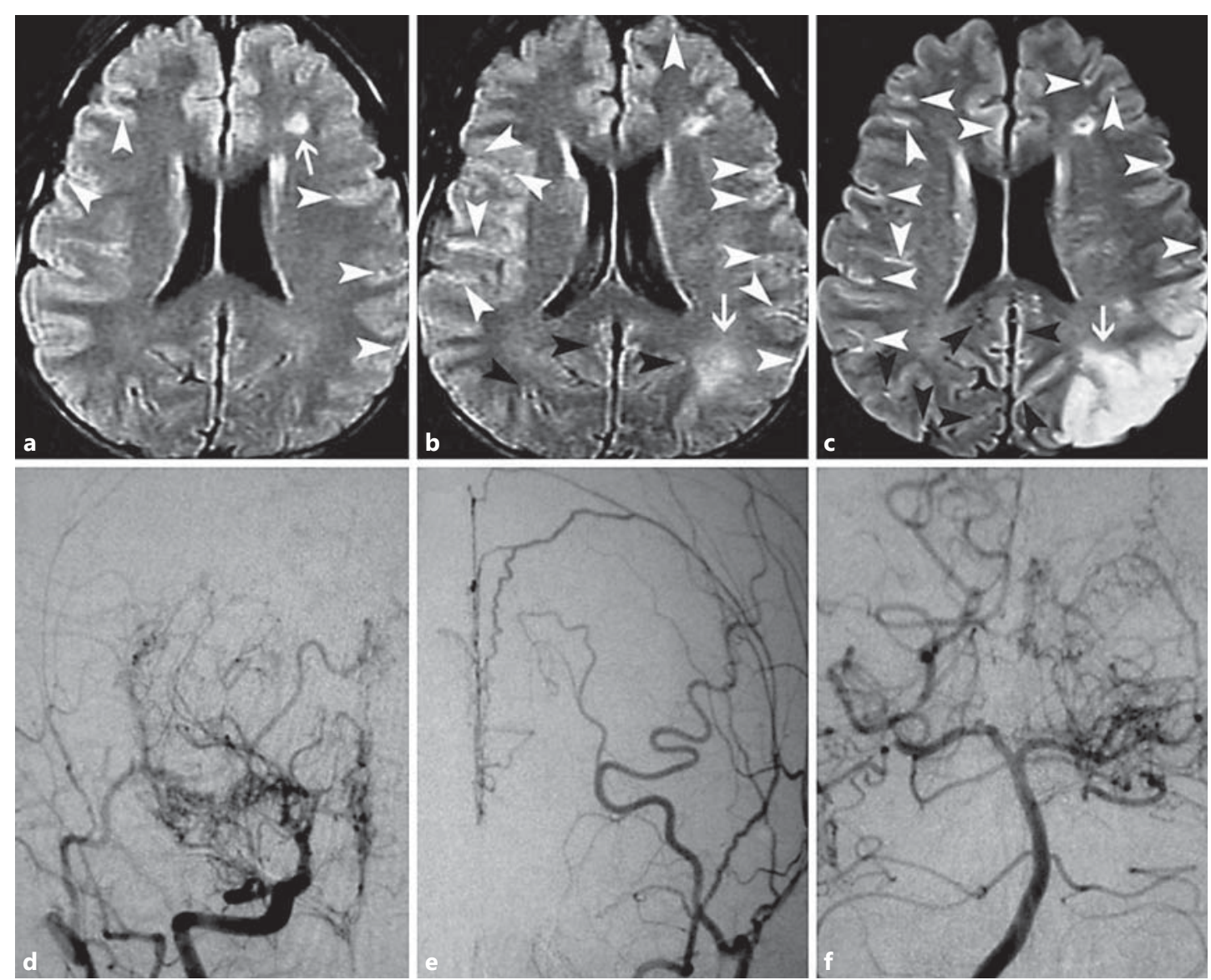

Fig. 4. Progression of HV on FLAIR-MRI and transformation of ischemic lesion patterns on axial FLAIR images in an MMD patient. a Initial HV locate in territories of the anterior cerebral circulation, especially in regions of the MCA (arrowheads). HV are not seen obviously in PCA territories. There is a small subcortical infarct located in the territory of the anterior cerebral circulation (arrow). b Four months later, HV progression is prominent in territories of the anterior cerebral circulation (white arrowheads) compared with the initial HV image (a). A few HV are visible in territories of the PCA (black arrowheads). In addition, the subcortical infarct is transformed from the anterior to the posterior cerebral circulation (arrow). c Eight months later, many new HV locate in cerebral sulci or on the brain surface compared with previous images (a, b), being especially prominent in PCA territories (arrowheads). Moreover, the cerebral infarct pattern is shifted from the subcortical to the cortical area of the brain (arrow). d-f Bilateral carotid and right vertebral angiographies establish a diagnosis of MMD.

vasively detect steno-occlusive lesions of the intracerebral artery, e.g. in MMD (fig. 4) [10-13], and hyperacute stroke with occlusion of a major intracerebral artery [7, 9]. Our recent study showed that HV location was more predominant in the sylvian fissure than in the other MCA territory in patients with intracerebral arterial steno-occlusive lesions [14], which was consistent with previous studies [7-9, 15]. Some studies have postulated that the leptomeningeal collaterals may be the underlying structures for $\operatorname{HV}[7,9,12,13]$. Indeed, angiographically, our study demonstrated that the slow residual anterograde and/or leptomeningeal retrograde blood flows are the origin of HV (fig. 5) [14]. Moreover, we speculate that as MMD progresses, HV could extend from the anterior to the posterior territory of the cerebral circulation, which could be a predictor of stroke onset in the posterior cerebral circulation. Addi- 

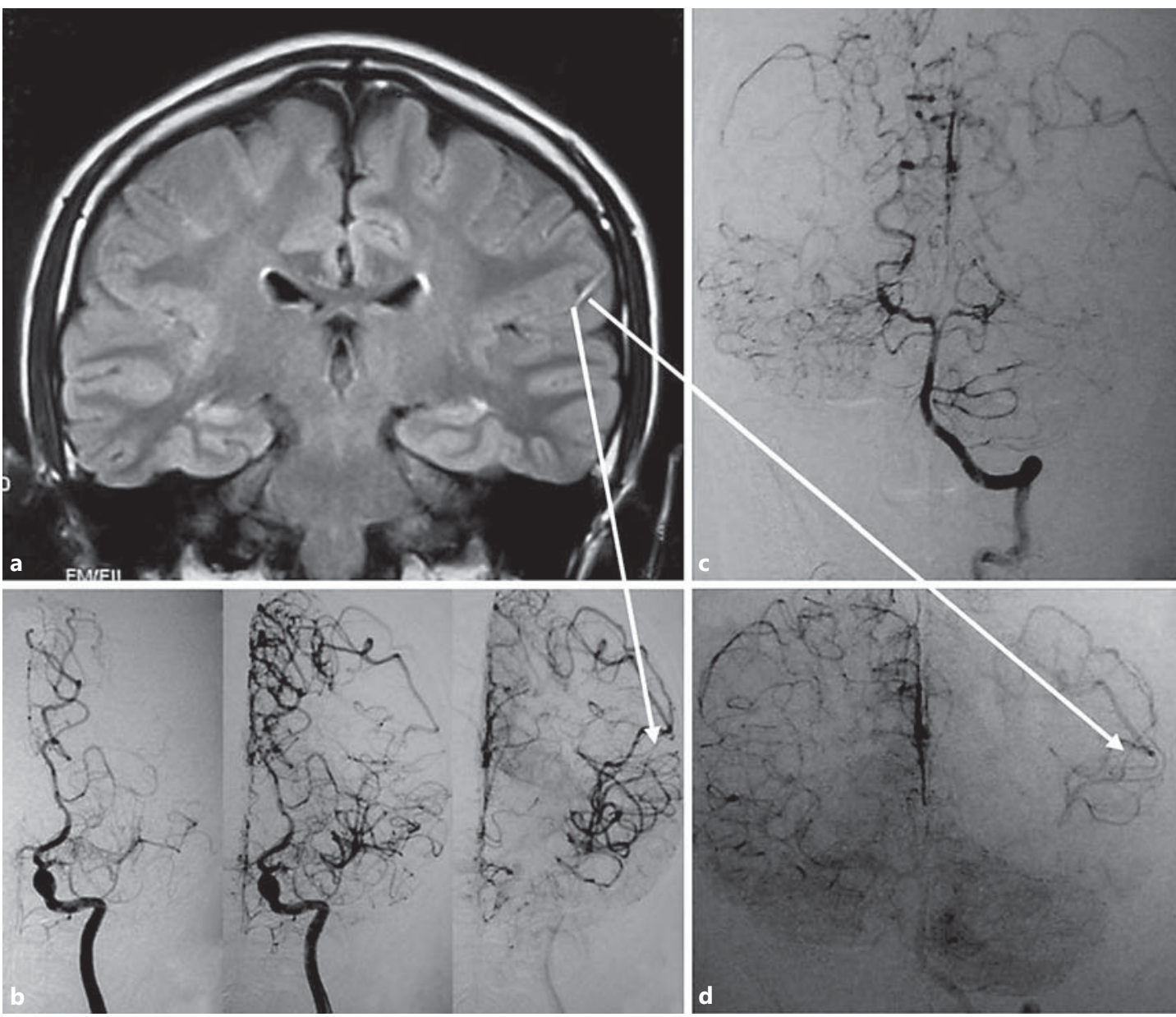

Fig. 5. Conventional cerebral angiography demonstrates that the slow leptomeningeal retrograde blood flows are the origin of HV in an MMD patient. a HV on FLAIR within the bilateral territories of the sylvian fissure and the cerebral sulci of the temporal-occipital junction (frontal lobe). b Serial arteriographic images from a selective left ICA injection show the retrograde collateral flow via leptomeningeal branches of the PCA, corresponding to HV within the left territory of the sylvian fissure (short arrow). c, d Serial left vertebral angiograms demonstrate the leptomeningeal collateral vessels filling retrogradely the distal MCA, similarly corresponding to HV within the left territory of the sylvian fissure (long arrow).

tionally, our results indicated that retrograde blood flow was more sluggish than anterograde flow, and it was commonly seen during the angiographic venous phase [16], which was consistent with a recent study [17]. Interestingly, our recent study implied that endovascular recanalization of ICA atherosclerotic occlusions resulted in a preoperative decrease in HV in the MCA territory, and a postoperative decrease in HV can be considered a marker for hemodynamic improvement [18].

\section{Computed Tomography Angiography}

CTA of the head and neck vessels has rapidly improved since helical CT was available to scan quickly and to capture the arterial phase of enhancement. The 64-row CT scanners facilitate isotropic imaging and provide high-resolution 3D reconstructions (fig. 6). This diagnostic modality is helpful for evaluating patients with suspected vascular abnormal- 

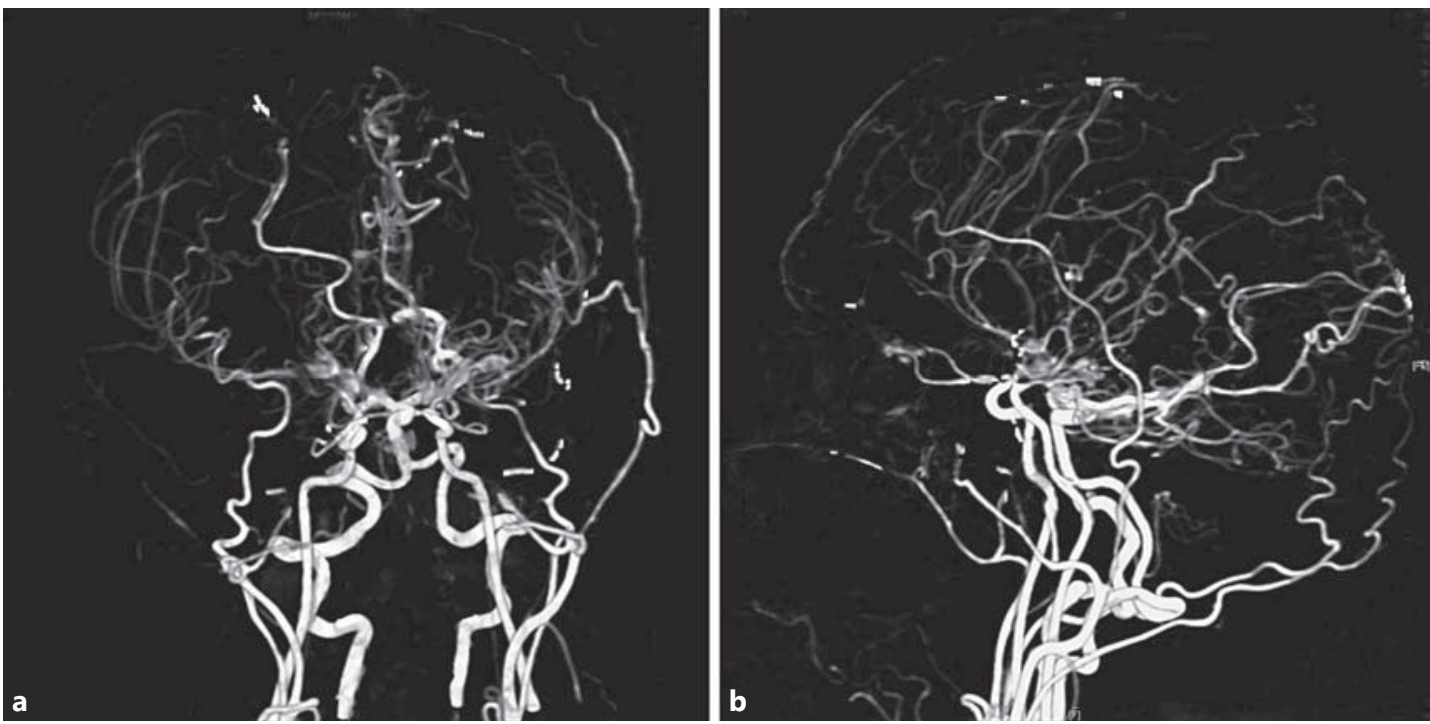

Fig. 6. A 3D reconstruction of a CT angiography showing an MMD patient.
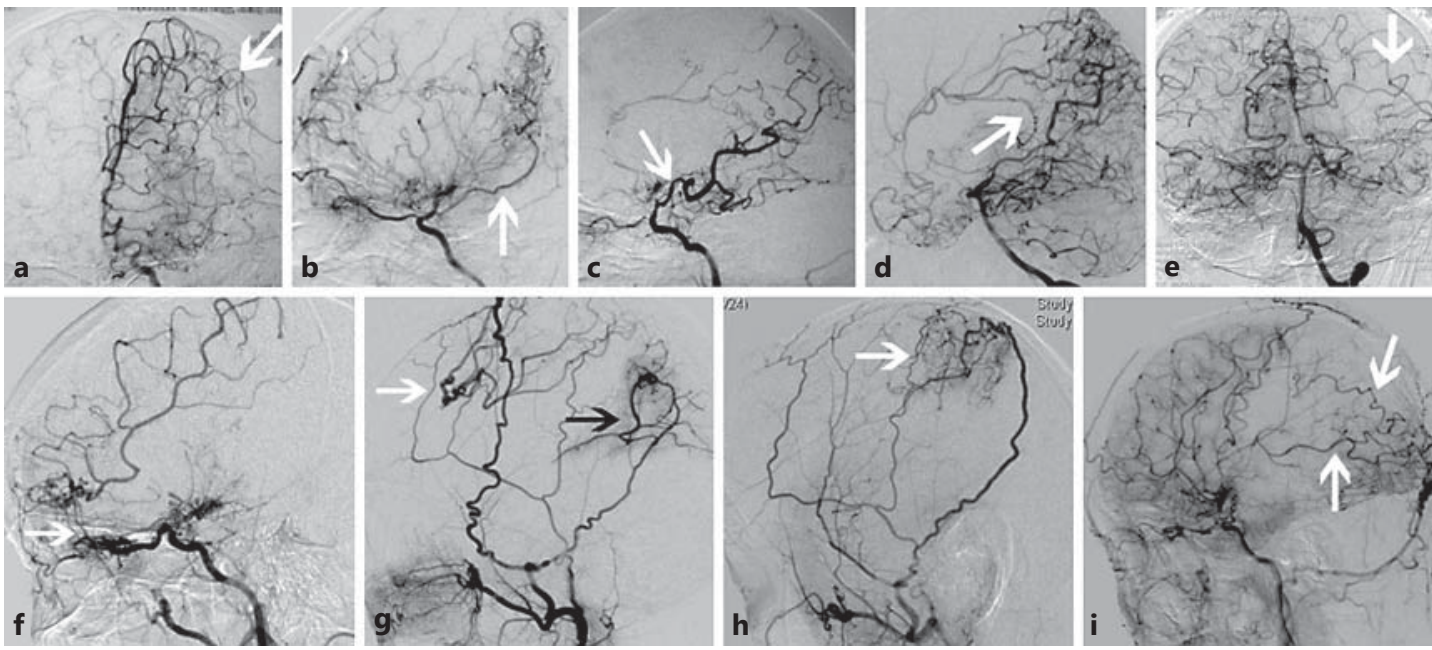

Fig. 7. Collateral patterns of MMD detected by conventional cerebral angiography. a ACA supplying the blood flow to the MCA territory via the MLA (arrow). b Dilation and extension of the AChA beyond the choroid fissure (arrow). c Patent PComA (arrow) providing blood flow to the territory of ACA and/or MCA via the PCA and MLA. $\mathbf{d}$ Blood flows from the posterior choroidal artery to the ACA territory via the posterior pericallosal artery (arrow). e Blood flows from the PCA to the ACA and/or MCA territory via the MLA (arrow). $\mathbf{f}$ Blood flows from the ophthalmic artery to the ACA territory via neovessels (arrow). $g$ Middle MLA supplying blood to the ACA (white arrow) and/or MCA (black arrow). $\mathbf{h}$ Superficial temporal artery supplying blood to the MCA (arrow). i Occipital artery supplying blood to the ACA and/or MCA (arrows). 
Fig. 8. Association of hemorrhagic stroke with high-grade AChAPComA. Patient 1: CT scan shows left intraventricular hemorrhage (a) and left ICA angiogram demonstrates grade 2 AChA-PComA (b). Patient 2: CT scan reveals temporal hemorrhage (c) and left ICA and vertebral artery angiograms show grade 2 AChA-PComA (d). Patient 3: CT scan reveals subarachnoid hemorrhage in the basal and ambient cistern (e); right vertebral artery angiogram demonstrates a large aneurysm (arrowhead) originating at the right bifurcation of the basilar artery (f), and right ICA angiogram shows grade 3 AChA-PComA (g).
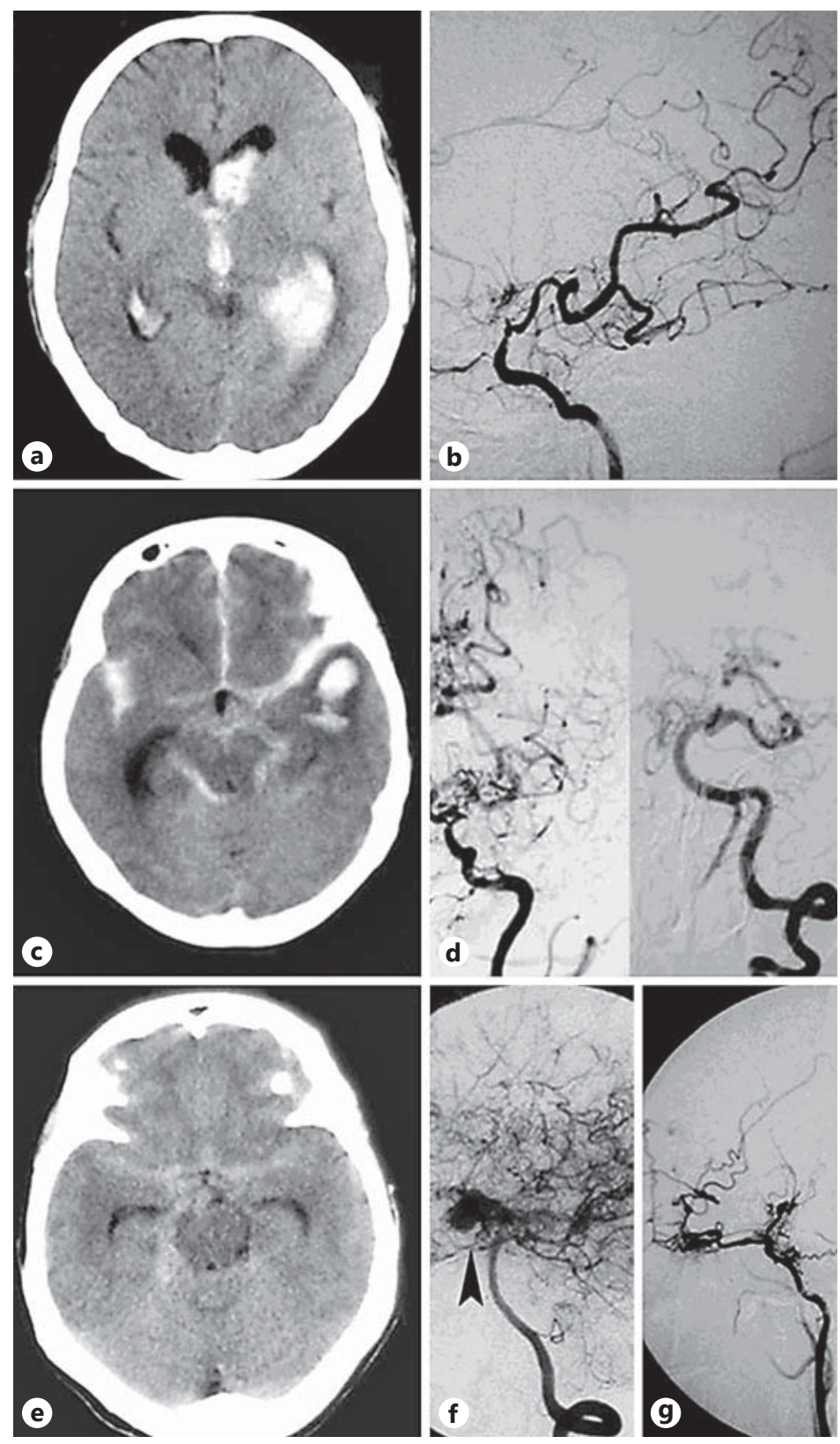

ities, particularly patients with moyamoya vessels. CTA is also useful for preoperative planning and for postsurgical verification of patency of an external carotid artery-ICA bypass [19].

\section{Collateral Circulation Patterns}

MMD presents the ultimate example of excessive collateralization over a chronic time course. Although the collateral circulation can be assessed by several methods, such as transcranial Doppler, CTA, MRA and digital subtraction angiography, the latter is still the gold standard. At present, large-sample studies investigating collateral patterns of MMD are scarce. 

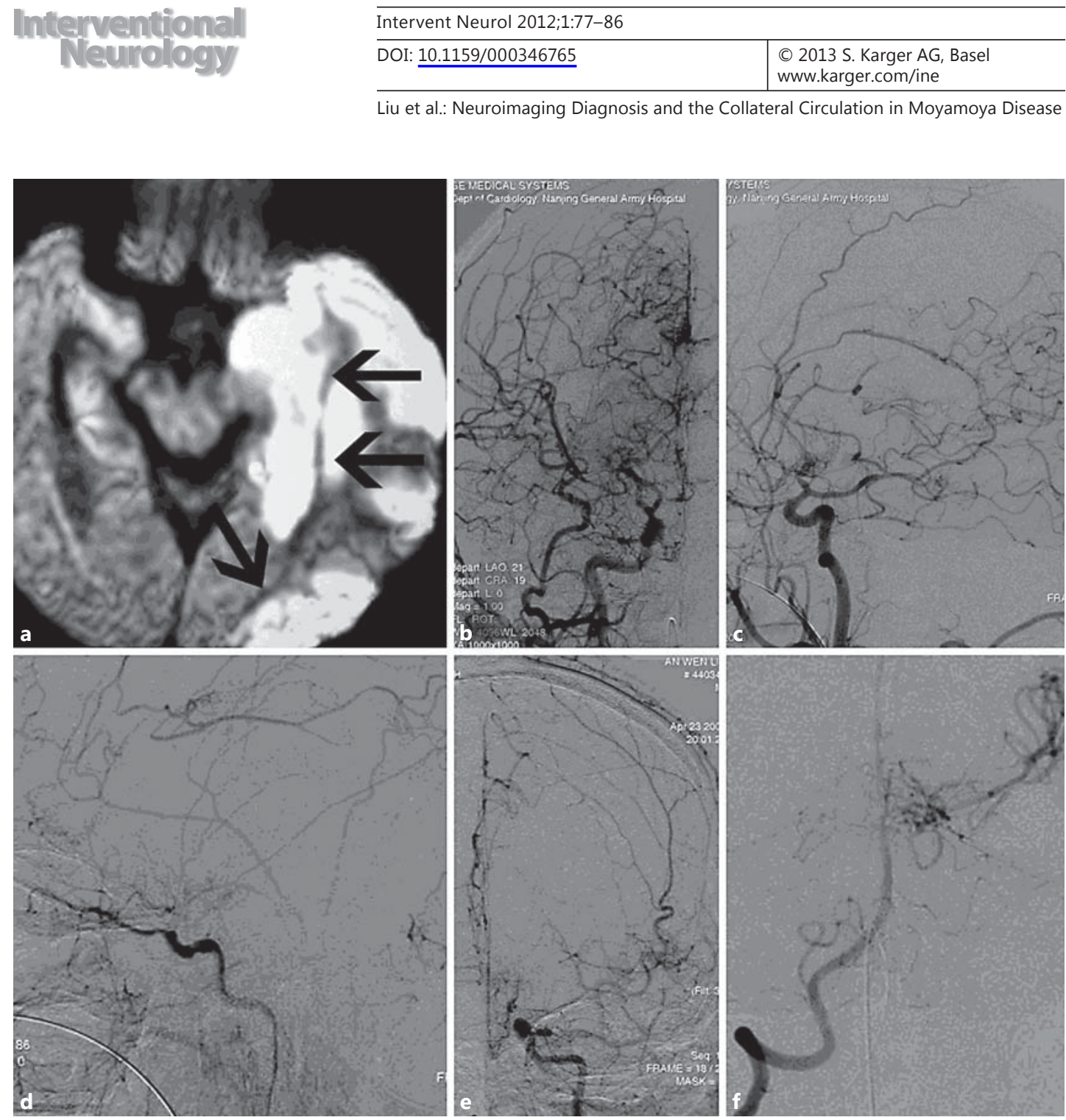

Fig. 9. Association of cerebral infarction with high-grade AChA-PComA. a Axial diffusion-weighted MR image demonstrating infarcted lesions in the territory of the left posterior circulation (arrows). b, c Right ICA angiograms disclose grade 2 AChA-PComA. d, e Left ICA angiograms show grade 3 AChA-PComA. $\mathbf{f}$ Left vertebral artery angiogram reveals collateral blood flow to the territory of the left MCA.

Therefore, elucidation of the collateral circulation patterns in MMD by conventional cerebral angiography is important. According to our recent study, there are 9 common collateral pathways in patients with MMD, including ACA $\rightarrow$ meningeal arteries (MLA) $\rightarrow$ MCA (fig. 7a), dilation and extension of the anterior choroidal artery beyond the choroid fissure (fig. $7 \mathrm{~b}$ ), the patent posterior communicating artery $\rightarrow$ the posterior cerebral artery (PCA) $\rightarrow$ MLA $\rightarrow$ ACA and/or MCA (fig. 7c), the posterior choroidal artery $\rightarrow$ the posterior pericallosal artery $\rightarrow$ ACA (fig. 7d), $\mathrm{PCA} \rightarrow$ MLA $\rightarrow$ ACA and/or MCA (fig. 7e), the ophthalmic artery $\rightarrow$ neovessels $\rightarrow$ ACA (fig. 7f), middle MLA $\rightarrow$ ACA and/or MCA (fig. 7g), superficial temporal artery $\rightarrow$ MCA (fig. 7h), and occipital artery $\rightarrow$ ACA and/or MCA (fig. 7i). In adults, the patterns of collateral distribution are changing with MMD progression. The collateral circulation, including dilation and extension of the anterior choroidal artery beyond the choroid fissure, patent posterior communicating artery $\rightarrow$ PCA $\rightarrow$ MLA $\rightarrow$ ACA and/or MCA, and posterior choroidal artery $\rightarrow$ posterior pericallosal arteries $\rightarrow$ ACA, accounted for a higher proportion, especially in the medium stage of disease duration, which suggested that these collaterals play an important role in blood flow recovery. 


\section{Stroke Patterns Related to the Collateral Circulation}

According to our recent data, the dilation and extension of anterior choroidal and posterior communicating arteries (AChA-PComA) are common patterns in the abovementioned collateral pathways in patients with MMD. Recently, changes in AChA-PComA were classified by conventional cerebral angiography into 4 different grades: grade 0 , normal; grade 1, dilation of the AChA within the choroidal fissure and/or dilation of the PComA without abnormally extensive branches; grade 2, dilation and extension of the AChA beyond the choroidal fissure and/or dilation of the PComA with abnormally extensive branches (posterior pericallosal arteries and/or leptomeningeal collateral vessels supplying the anterior cerebral circulation), and grade 3, disappearance of the AChA-PComA (the occlusion is situated proximal to the PComA of the ICA) [2]. This study showed that stroke patterns were related to angiographic changes in the AChA-PComA in adult MMD patients. For lowgrade AChA-PComA (including grades 0 and 1), the proportion of ischemic lesions was similar to the proportion of hemorrhagic lesions involving mainly the anterior circulation. Grade 2 was predominantly associated with intraventricular (fig. 8a) and temporal hemorrhage (fig. 8c), and grade 3 was predominantly correlated with aneurysmal subarachnoid hemorrhage (fig. 8e) and cerebral infarction (fig. 9a) in the territory of the posterior circulation. This is the first report on angiographic changes in AChA-PComA. Moreover, our results indicated that grades 0 and 1 are considered the early stages of MMD, while grades 2 and 3 reflect the late stages of MMD. The angiographic characteristics of AChA-PComA may predict the onset of certain stroke patterns in adult MMD.

\section{Disclosure Statement}

I declare that I have no conflicts of interest regarding funding arrangement related to my research.

\section{References}

1 Suzuki J, Takaku A: Cerebrovascular 'moyamoya' disease. Disease showing abnormal net-like vessels in base of brain. Arch Neurol 1969;20:288-299.

2 Liu W, Zhu S, Wang X, Yue X, Zhou Zhi, Wang H, Xu G, Zhou C, Liu X: Evaluation of angiographic changes of the anterior choroidal and posterior communicating arteries for predicting cerebrovascular lesions in adult moyamoya disease. J Clin Neurosci 2011;18:374-378.

3 Harada A, Fujii Y, Yoneoka Y, Takeuchi S, Tanaka R, Nakada T: High-field magnetic resonance imaging in patients with moyamoya disease. J Neurosurg 2001;94:233-237.

- 4 Fukui M: Guidelines for the diagnosis and treatment of spontaneous occlusion of the circle of Willis ('moyamoya' disease). Research Committee on Spontaneous Occlusion of the Circle of Willis (Moyamoya Disease) of the Ministry of Health and Welfare, Japan. Clin Neurol Neurosurg 1997;99(suppl 2):S238-S240.

5 Houkin K, Aoki T, Takahashi A, Abe H: Diagnosis of moyamoya disease with magnetic resonance angiography. Stroke 1994;25:2159-2164.

- 6 Yamada I, Suzuki S, Matsushima Y: Moyamoya disease: comparison of assessment with MR angiography and MR imaging versus conventional angiography. Radiology 1995;196:211-218.

- 7 Lee KY, Latour LL, Luby M, Hsia AW, Merino JG, Warach S: Distal hyperintense vessels on FLAIR: an MRI marker for collateral circulation in acute stroke? Neurology 2009;72:1134-1139.

8 Iancu-Gontard D, Oppenheim C, Touzé E, Méary E, Zuber M, Mas JL, Frédy D, Meder JF: Evaluation of hyperintense vessels on FLAIR MRI for the diagnosis of multiple intracerebral arterial stenoses. Stroke 2003;34: 1886-1891.

9 Kamran S, Bates V, Bakshi R, Wright P, Kinkel W, Miletich R: Significance of hyperintense vessels on FLAIR MRI in acute stroke. Neurology 2000;55:265-269.

10 Marshall S, Hawley JS, Nyquist PA, Degraba T: The 'ivy sign' of adult moyamoya disease. Neurologist 2009;15: 367-368. 
11 Mori N, Mugikura S, Higano S, Kaneta T, Fujimura M, Umetsu A, Murata T, Takahashi S: The leptomeningeal 'ivy sign' on fluid-attenuated inversion recovery MR imaging in moyamoya disease: a sign of decreased cerebral vascular reserve? AJNR Am J Neuroradiol 2009;30:930-935.

$\checkmark 12$ Fujiwara H, Momoshima S, Kuribayashi S: Leptomeningeal high signal intensity (ivy sign) on fluid-attenuated inversion-recovery (FLAIR) MR images in moyamoya disease. Eur J Radiol 2005;55:224-230.

13 Yoon HK, Shin HJ, Chang YW: 'Ivy sign' in childhood moyamoya disease: depiction on FLAIR and contrastenhanced T1-weighted MR images. Radiology 2002;223:384-389.

14 Liu W, Xu G, Yue X, Wang X, Ma M, Zhang R, Wang H, Zhou C, Liu X: Hyperintense vessels on FLAIR: a useful non-invasive method for assessing intracerebral collaterals. Eur J Radiol 2011;80:786-791.

15 Sanossian N, Saver JL, Alger JR, Kim D, Duckwiler GR, Jahan R, Vinuela F, Ovbiagele B, Liebeskind DS: Angiography reveals that fluid-attenuated inversion recovery vascular hyperintensities are due to slow flow, not thrombus. AJNR Am J Neuroradiol 2009;30:564-568.

16 Calabrese LH, Gragg LA, Furlan AJ: Benign angiopathy: a distinct subset of angiographically defined primary angiitis of the central nervous system. J Rheumatol 1993;20:2046-2050.

17 Kawashima M, Noguchi T, Takase Y, Nakahara Y, Matsushima T: Decrease in leptomeningeal ivy sign on fluidattenuated inversion recovery images after cerebral revascularization in patients with moyamoya disease. AJNR Am J Neuroradiol 2010;31:1713-1718.

18 Liu W, Yin Q, Yao L, Zhu S, Xu G, Zhang R, Ke K, Liu X: Decreased hyperintense vessels on FLAIR images after endovascular recanalization of symptomatic internal carotid artery occlusion. Eur J Radiol 2012;81:15951600.

19 Coppens JR, Cantando JD, Abdulrauf SI: Minimally invasive superficial temporal artery to middle cerebral artery bypass through an enlarged bur hole: the use of computed tomography angiography neuronavigation in surgical planning. J Neurosurg 2008;109:553-558. 